\title{
Comparison of the 'glory' with coherent backscattering of light in turbid media
}

\author{
Ralf Lenke $^{1,2}$, Ulirich Mack ${ }^{1}$ and Greorg Maret ${ }^{1}$ \\ ${ }^{1}$ University of Konstanz, Box 5560, D-78457 Konstanz, Germany \\ ${ }^{2}$ Institut Charles Sadron, 6 rue Boussingault, F-67083 Strasbourg Cedex, France
}

\begin{abstract}
The 'glory' results from light scattering of single, sub-millimetre to millimetre-sized spheres in the exact backscattering direction. The so-called coherent backscattering by disordered media is the intensity enhancement in the very same direction due to the interference between each light path and its reversed path. This pair of paths always exists in multiple-scattering media. The two phenomena have very similar properties, which will be studied and compared in more detail, experimentally as well as by numerical calculations.
\end{abstract}

Keywords: Mie theory of large spheres, multiple scattering of light

(Some figures in this article are in colour only in the electronic version)

\section{Introduction}

The 'glory' is a natural light scattering phenomenon which can be observed around one's own shadow from sunlight falling on clouds or fog. Around the shadow one can observe bright, coloured rings, which in the ideal case reproduce at regular distances up to about five times (see figure 1). This phenomenon is a light scattering effect of the individual water droplets in the clouds resulting from the rotational symmetry of the droplets around the optical axis. Due to this symmetry, there are whole classes of light rays in the sphere having exactly the same length and thus interfering constructively in the exact backscattering direction (so-called axial focusing [1]). Unlike the rainbow, the 'glory' is an interference effect ${ }^{3}$. The aperture angle of the rings is proportional to the wavelength in air $\lambda_{0}$ and inversely proportional to the radius $a$ of the droplets. Of course, the perceived diameter of the rings is independent of the distance from the clouds. The diameter of water droplets in clouds range from some micrometres to some tens of micrometres, normally. The particle size distribution, however, mostly fluctuates only by a few per cent. Nice observations of the 'glory' in nature were reported for droplet diameters of about $40 \mu \mathrm{m}$ [2], for example. Note that the 'glory' is not only restricted to water droplets but is generated by any transparent sphere with diameters between some micrometres and millimetres.

3 Of course, there are also interference effects which accompany the rainbow, for example the supernumerary arcs. However, they are relatively faint.

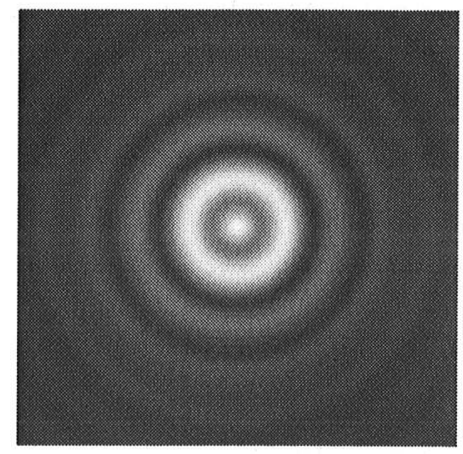

Figure 1. Phenomenon of the 'glory' calculated using Mie theory for water droplets with a diameter of $20 \mu \mathrm{m}$. Angle range: $\pm 7.45^{\circ}$ around the exact backscattering direction. In nature, the bright spot in the centre is covered by one's own shadow and the coloured rings are superimposed on a white diffuse background originating from the light which was scattered several times in the cloud.

There are other natural phenomena resulting in an increased backscattering intensity [3]: the cornfield effect, the Heiligenschein and the sylvanshine. The cornfield effect originates from the simple geometrical fact that the shadow of each blade is hidden by the blade itself, thus enhancing the average intensity in the exact backscattering direction. The Heiligenschein and the sylvanshine result from lens effects (cat's eye) of dewdrops on a meadow or on leaves of special plants, respectively. These geometrical effects are not studied 
further here. They can be brighter than the 'glory' but they are always colourless and do not show any oscillatory signature.

If the spheres are not perfectly round, i.e. if the length of the light paths belonging to one ray class differ by about $\lambda$, the rotational invariance is lost. However, the symmetry between each light path and its reversed path is independent of any deformation of the sphere, still leading to a twofold relative intensity enhancement in the exact backscattering direction. For reasons which will be discussed further below with respect to light scattering by disordered media, we call this interference between a pair of paths coherent backscattering (CB). The backscattering pattern of monodisperse but imperfect spheres resembles to that of the 'glory'. The similarities and differences will be studied in more detail in this paper.

If, in addition, the imperfect spheres are polydisperse, the oscillations in the backscattering profile are averaged out and only the intensity enhancement due to $\mathrm{CB}$ is left. The light scattering properties of such samples are very similar to that of completely random, disordered media such as milk, snow or any white paint. In these materials, this intensity enhancement is known as the $C B$ cone [4,5]. In a semi-infinite, non-absorbing turbid sample the $\mathrm{CB}$ cone has a triangular tip and a full width at half maximum of about $1 / k_{0} \ell^{*}$ radians; $k_{0}=2 \pi / \lambda_{0}$ is the wavenumber in air. The so-called transport mean free path $\ell^{\star}$. is the characteristic length of the photon random walk in the disordered system; $1 / \ell^{\star}$ quantifies the turbidity of the medium.

Precisely speaking, the twofold CB intensity enhancement of a disordered medium is only observed if incident and detected polarization states are the same. This is guaranteed by the theorem of reciprocity [6], which states that the scattering matrices describing any direct and reversed light path are the transpose of each other. In the crossed channels, a cone is only observed to the extent that the scattering matrices are symmetric [7]. In turbid media, the enhancement factor in the crossed channels is smaller than 1.2, generally. For a perfect single sphere, the matrix is always symmetric.

In the course of this discussion, we tacitly made the transition from multiple scattering of light rays in single particles, using a ray optics approach, to multiple light scattering between different particles. In dense media the distinction between the two regimes cannot always be made as easily as for clouds, for example, where the 'glory' is a singleand the white background a many-particle effect. In clouds, the distance $\ell^{\star}$, which is about the visibility distance, is orders of magnitudes larger than the water droplet diameter. For a detailed description of the phenomenon of $\mathrm{CB}$ in disordered media the reader is referred to [7]. In the following we restrict ourselves to a comparison between the 'glory' and CB of imperfect spheres.

\section{The 'glory'}

Mie theory [8] is an exact analytic solution of Maxwell's equations for light scattering by spheres with a uniform index of refraction. However, this is not a trivial solution but a row expansion of spherical Bessel functions. A vast literature of efforts exists to explain natural optical phenomena of water droplets by more 'hand-waving' arguments (see e.g. [1,8] and references therein). These explanations, mostly making use

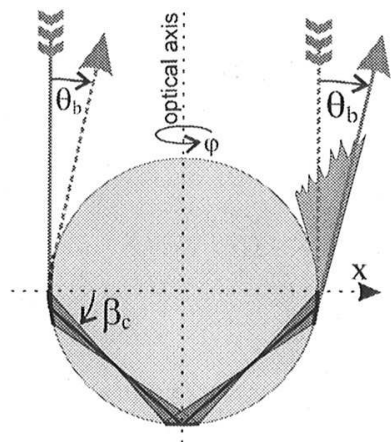

Figure 2. Illustration of light rays with one reflection and surface wave part in a water droplet. Any path inside the marked region is possible, all having the same optical length. $\beta_{c}$ is the critical angle of total reflection.

of ray optics, are more satisfying. Moreover, they enable the comparison with other phenomena such as $\mathrm{CB}$ and they have helped in developing powerful calculation algorithms. The validity of the ray optics approach for spheres larger than several $\lambda_{0}$ is consolidated by the expansion of Mie's solution in the so-called Debye terms [1,9]. The Mie terms correspond to partial waves with origin in the centre of the sphere. Debye's row expansion decomposes each Mie term in waves, which are reflected by or pass through the surface of the sphere according to Fresnel's law. The reflected waves run through the origin again and can be reflected a second time and so on. A Debye term is obtained by collecting all the terms with the same number of reflections. In most cases there is only one possibility for a light ray with a given number of reflections inside the sphere, thus allowing a direct comparison between ray optics and Debye terms.

For water droplets (more precisely spheres with a relative index of refraction $m<\sqrt{ } 2$ ), there are no 'trivial' light rays corresponding to scattering in the exact backward direction after one reflection inside the sphere. There are only edge rays, entering the sphere under the critical angle of total reflection. which in addition have propagated $14.4^{\circ}$ (for $m=1.333$ ) along the surface in total (see figure 2). For a detailed description of those surface waves see $[1,8]$. Other rays with more than one reflection inside the sphere are possible as well. However, with increasing number of reflections, again only the edge rays are of importance as only in this case (which is close to total reflection) is the remaining amount of the repeatedly reflected intensity non-negligible [1]. It turns out that light rays with ten internal reflections form the next important term for the natural 'glory'. After averaging the size parameter $k_{0} a$ over $\pm 5 \%$ ( $m=1.333$, no absorption), we found that the term with ten reflections predominates over the one-reflection term for values $k_{\mathrm{o}} a \gtrsim 175$. For values $k_{\mathrm{o}} a \lesssim 1200$, both terms together contribute $75 \%$ to the totally reflected intensity (where it makes no difference whether the terms are added coherently or incoherently $)^{4}$. The absolute amount of backscattered intensity is very sensitive to the precise values of $k_{0} a$ and $m$. We shall not discuss this further here as for the phenomenon of the 'glory', i.e. a relative intensity enhancement in the exact backscattering direction with respect to somewhat larger backscattering

4 All the calculations have been performed using the Mie Scattering User Interface (Valley Scientific 1998). 


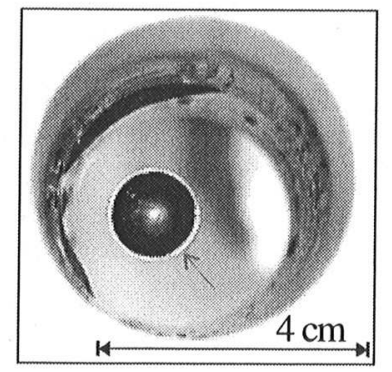

Figure 3. Magnetically levitated water drop in the vertical bore of a superconducting magnet. Field strength $18 \mathrm{~T}$. Diameter approximately $1.3 \mathrm{~cm}$. The flashlight of the camera scattered back via edge rays (arrow) is clearly visible.

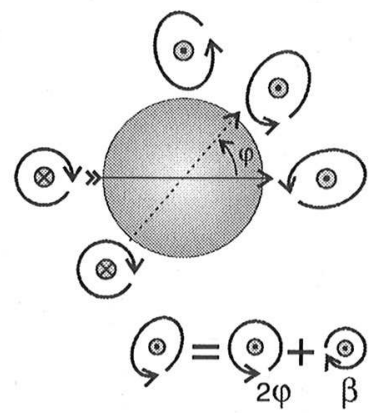

Figure 4. Illustration of the polarization of rays backscattered from different points on a sphere in the case of circular polarized incident light. By decomposition into the linear polarized eigenstates, this drawing also illustrates that the detected intensity in the case of linear polarization is $\varphi$ dependent for $\theta_{b}>0$, generally [1]

angles, it is only necessary that light is scattered in the backward direction at all. The absolute amount of backscattered intensity is only of secondary importance in this context. The predominant contribution of the edge rays accounts for the strong coloured regular 'glory' rings corresponding to the interference pattern of a light ring. On the photograph in figure 3 the edge rays of a magnetically levitated water drop are clearly visible. Magnetic levitation is quite a promising method to study water droplets of nearly any size under 'micro-gravity'. Other techniques, such as suspended [10], free falling [11] or optically levitated [12] droplets, have the shortcomings of deforming the sphere or being restricted to very small particles. Unfortunately, this $20 \mathrm{~T}$ magnet was available only for a short time, so we cannot present further measurements of magnetically levitated spheres here.

In the following we shall only study circular polarized light, which better reflects the rotational invariance of the problem. Moreover, the case of the same incident and detected circular polarization, ' ++ ', is preferred for CB measurements, as then direct reflexions and single scattering, which do not contribute to $\mathrm{CB}$, are suppressed. Figure 4 illustrates the polarization of the edge rays (circular incidence) scattered in the exact backscattering direction. In the most general case the scattered light is elliptically polarized. For symmetry reasons the ellipse is rotated by the azimuthal angle $\varphi$ along the contour of the sphere. By an appropriate choice of the coordinate system of each ray one can easily show that these ellipses can be decomposed into a part with the same circular polarization as incident but with a relative phase shift of $2 \varphi$ and

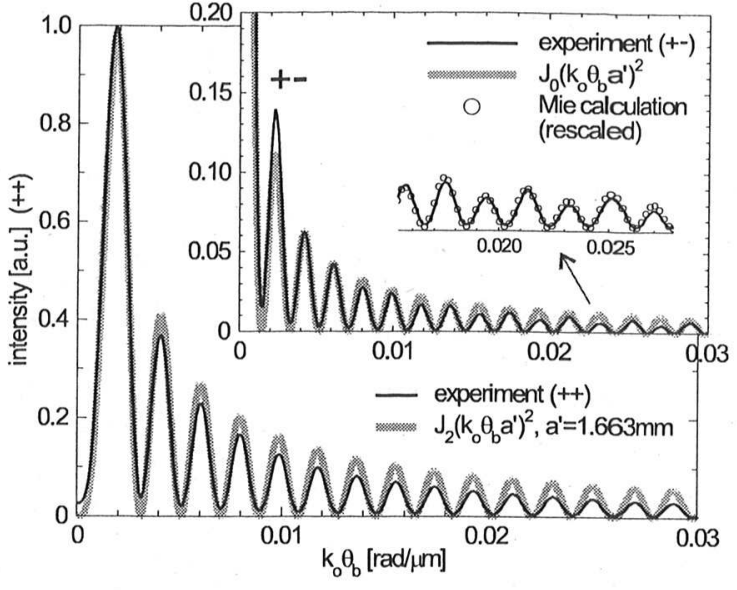

Figure 5. Measured intensity distribution of a glass sphere $\left(m=1.8625+\mathrm{i} 8 \times 10^{-7}\right.$, diameter $\left.5000 \pm 1 \mu \mathrm{m}\right)$ as a function of backscattering angle $\theta_{b}$. Plane-parallel incident wave with $\lambda_{\mathrm{o}}=514.5 \mathrm{~nm}$, circular polarization. The sphere floated on an air jet. The square of the second-order Bessel function well reflects the scattering profile of the case ' ++ '. Here, the effective radius $a$ ' is not the sphere radius, i.e. does not correspond to edge rays as $m>\sqrt{ } 2$, but [8] $a^{\prime} / a=m \sqrt{ } 1-m^{2} / 4$ (minus an error of $2 \%$ ). Inset: the same measurements for the case ' +- ' and comparison with the zeroth-order Bessel function. The curves are rescaled to unity for $\theta_{b}=0$. The measured and calculated scattering patterns do not precisely correspond to $J_{0}^{2}$ but also contain other frequency parts as can be seen from the blown-up segment. The experimental set-up is described in section 4 .

the opposite state with a $\varphi$-independent constant phase shift $\beta$. Phase-consistent integration over all edge rays results, in the case ' ++ ', in an angular intensity distribution proportional to $J_{2}\left(k_{\mathrm{o}} a \theta_{b}\right)^{2}$ (see figure 5), where $\theta_{b}$ is the angle with respect to the exact backscattering direction. $J_{2}$ is the Bessel function of second order, whose value is zero for $\theta_{b}=0$. In the case of orthogonal incident and detected circular polarization ' +- ', the square of a zeroth-order Bessel function $J_{0}\left(k_{0} a \theta_{b}\right)^{2}$ is obtained (see the inset of figure 5).

Such simple Bessel functions are generally not obtained from exact Mie calculations for specific values of $k_{0} a$ and $m$, especially not in the case '+-'. For example, Mie calculations for the case ' +- ' in figure 5 reveal an additional oscillating term, which we could also detect in our measurements. For smaller particles, the situation is even worse and the scattering pattern can completely differ from $J_{0}^{2}$. The case ' ++ ' is generally approximated quite well by $J_{2}^{2}$. In any case, after averaging over a certain parameter range both Bessel functions well reflect the scattering patterns, as exemplified in figure 7 for the 'glory' of water droplets with a diameter of $20 \mu \mathrm{m}$ (for comparison, the inset of figure 8 shows the scattering pattern for one specific parameter set). Note that the 'effective radius' in the fitted Bessel functions is about $3 \%$ larger than the real radius of the water droplets. This can be explained by the fact that for $m<\sqrt{ } 2$ the light is scattered in and around the exact backscattering direction via surface waves (see figure 2). Therefore, one can suppose that the scattering patterns are better approximated by $J_{0 / 2}\left(k_{s} a \theta_{b}\right)^{2}$, where $k_{s}$ is the surface wavenumber. For this case, we obtain $[1,11] k_{s} / k_{0} \approx 1.036$.

Figure 8 shows the calculated scattering patterns for a $20 \mu \mathrm{m}$ droplet averaged over the red, green and blue spectrum range for non-polarized light. These curves have 


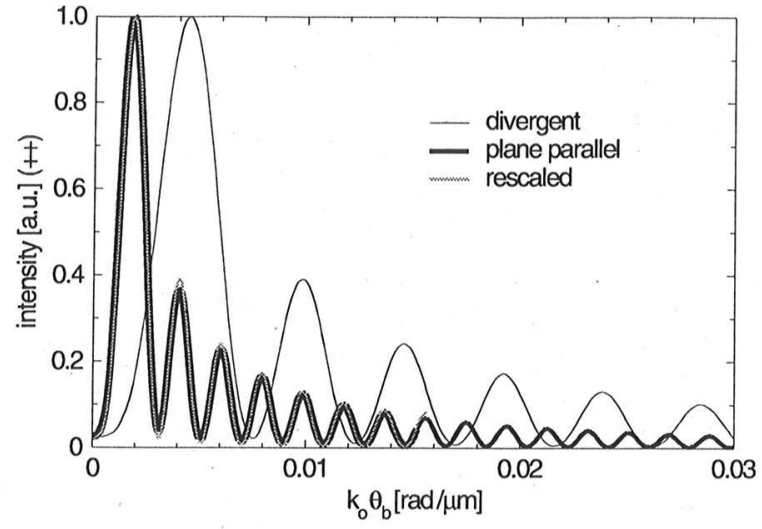

Figure 6. Similar measurement as in figure 5 but with illumination by a divergent light source. After linear rescaling in the ordinate according to [7], the scattering pattern is identical to that with a plane-parallel illumination.

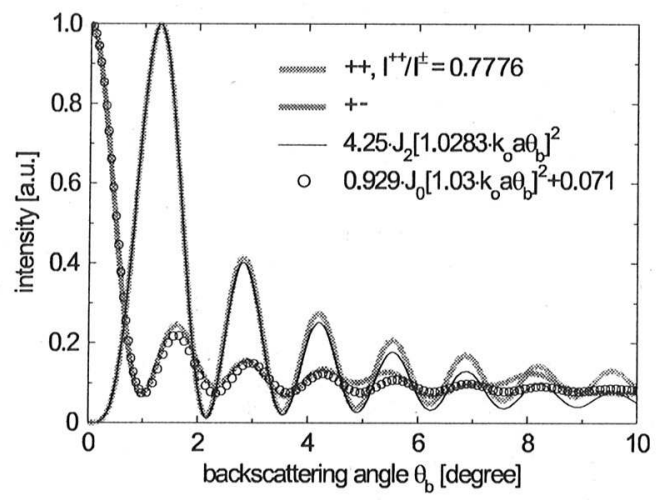

Figure 7. Mie calculations for a water droplet ( $m=1.333$, no absorption, diameter $20 \mu \mathrm{m}$ ) in backscattering direction for circular polarized light. The wavelength was averaged between 0.45 and $0.5 \mu \mathrm{m}$. The intensity of the case '+-' was rescaled by a factor 0.7776. The Bessel functions were averaged over the same wavelength range. An 'incoherent' part of 0.071 had to be added to $J_{0}^{2}$. This results from the fact that in the case ' +- ' the scattering pattern for one specific parameter set still contains other, non-trivial oscillating terms, which disappear only on average. Those additional oscillating terms, for which we do not have any simple explanation, essentially originate from the Debye term corresponding to one reflection inside the sphere.

been used to create figure 1. Of course, in order to numerically reproduce the natural 'glory', one should also take into account the spectrum of the sun, the sensitivity of the eye and the chromatographic reproduction on a screen or a photograph [13-15], as well as the droplet size distribution and the divergence of the sunlight (or equivalently its coherence area) [16]. Sunlight on earth is to $50 \%$ coherent within a region of $(40 \mu \mathrm{m})^{2}$ [17], giving approximately the upper limit of the droplet diameter for the 'glory'. Here, our intention was only to give an idea of the natural 'glory'. The result is quite satisfying compared with [2], for example.

\section{Coherent backscattering}

So far, we have only considered perfect spheres. Figure 9 shows a measurement similar to that of figure 5 but for a sample of imperfect glass beads in water. The scattering

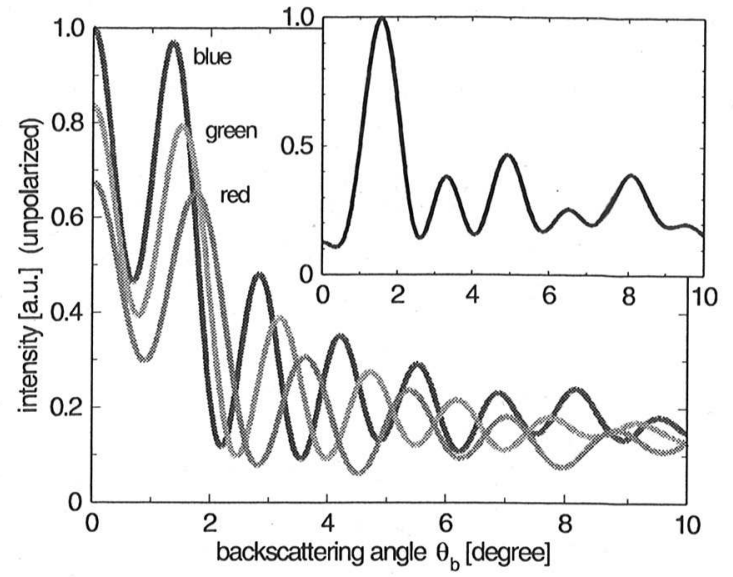

Figure 8. Backscattered intensity (unpolarized) of a water droplet (diameter $=20 \mu \mathrm{m}$ ) averaged over $0.45-0.5 \mu \mathrm{m}, 0.49-0.58 \mu \mathrm{m}$ and $0.57-0.65 \mu \mathrm{m}$. Inset: the same droplet but at a fixed wavelength of $\lambda_{\mathrm{o}}=0.56 \mu \mathrm{m}$.

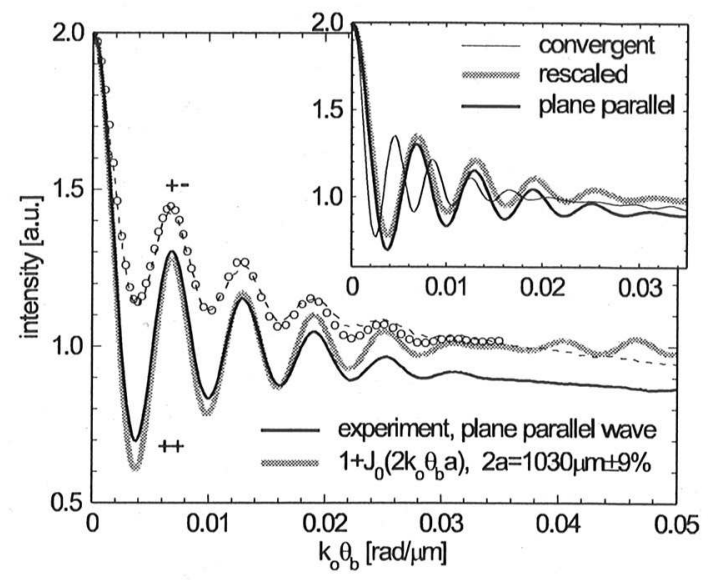

Figure 9. Measured backscattering intensity for a sample of imperfect glass beads in water and comparison with the zeroth-order Bessel function. Plane-parallel incident wave, circular polarization, $m=1.5 / 1.333, \lambda=514.5 \mathrm{~nm}$. $J_{0}$ was averaged in the particle diameter over $\pm 9 \%$. The fitted average diameter was a little smaller than the value given by the manufacturer $(1090 \mu \mathrm{m} \pm 9 \%)$. Dashed curve: measured crossed circular polarized channel. For comparison, the curve $1+J_{0}$ was rescaled according to the transformation $y=(y-1) * 0.55+1.45-26 * x+390 * x^{2}$ (points), which corresponds to a CB enhancement of only $2 / 1.45=1.38$. Inset: measurement with a convergent incident wave and comparison (by rescaling [7]) with the measurement with a plane-parallel incident wave.

pattern for the case ' ++ ' is well approached by $1+J_{0}\left(2 k_{0} a \theta_{b}\right)$. Such behaviour is obtained when there is only a constructive interference between each light path and its reversed path. Light paths with different azimuthal angles $\varphi$ (see figure 4) are added incoherently. This situation is analogous to that of CB in multiple-scattering media. In the crossed channel '+-' essentially the same scattering pattern is found but with a lower contrast, which suggests that the scattering matrices of the light rays are no longer completely symmetric.

The phenomenon of coherent backscattering is also called weak localization as it sends more light back to the origin of the light source than a model neglecting interference effects. In a sense, $\mathrm{CB}$ acts like a phase conjugating mirror: when 


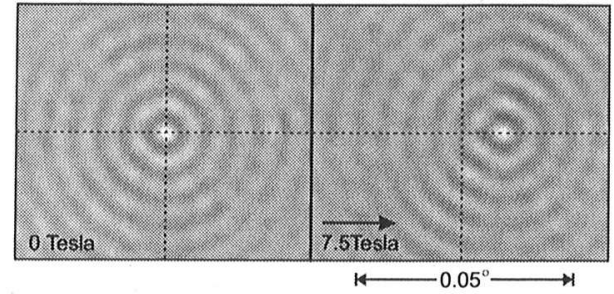

Figure 10. Influence of a magnetic field on the backscattering pattern of a glass sphere with a specific Verdet constant of $V=-166 \mathrm{rad} \mathrm{T} \mathrm{m}^{-1}$ at $\lambda_{\mathrm{o}}=457.9 \mathrm{~nm}$ (temperature $15^{\circ} \mathrm{C}$ ). Diameter $5.2 \mathrm{~mm}, m=1.7$, material 'Hoya FR5'. At $7.5 \mathrm{~T}$ the whole diffraction pattern was shifted in the direction of the magnetic field by an angle of $1.6 \times 10^{-4} \mathrm{rad}$.

using divergent or convergent light, the focus of the diffraction pattern lies in a plane through the (image) light source. The same is true for the 'glory'. Of course, the diffraction pattern of one single sphere can be detected at any point along the optical axis. However, in the case of many spheres or a moving sphere a sharp diffraction pattern is only obtained at the intersection of all optical axes. We have verified this behaviour for the 'glory' (see figure 6) and for CB in the case of imperfect spheres (inset of figure 9). Similar experiments for the $\mathrm{CB}$ cone of disordered media can be found in [7] and references there.

The only effect which destroys CB is Faraday rotation [18]. However, this is only if the light is also depolarized by the multiple scattering [19]. For the part of the light where the polarization is preserved, the $\mathrm{CB}$ cone is not destroyed but shifted out of the exact backscattering direction when applying a magnetic field perpendicular to the incident circularly polarized light $[19,20]$. We could also observe this Faraday-induced shift for the backscattering pattern of glass spheres with a high Verdet constant (see figure 10). Experimentally, we verified a linear shift corresponding to an angle of $-2 \sigma V \vec{B} / k_{0}$ in the direction of the magnetic field $\vec{B}$ ( $\sigma$ is the handedness of the circular polarization), once for a lead glass sphere (Schott SF57, diameter $5 \mathrm{~mm}$, index of refraction $m=1.88$ ) with a specific Verdet constant of $V=49 \operatorname{rad~} \mathrm{T} \mathrm{m}^{-1}$ and another time for a sphere made of a paramagnetic glass containing about $50 \mathrm{wt} \% \mathrm{~Tb}^{3+}$ ions (see figure 10). No shift was observed in the case ' +- ', as expected [19]. These spheres only showed a backscattering pattern similar to that of figure 9 (imperfect spheres), but there is no reason why this shift should not take place for perfect spheres as well. This behaviour under magnetic field nicely demonstrates that CB and the 'glory' are interference effects.

\section{Experimental set-up}

For these experiments we used the 'standard' CB set-up described in [7, 19], for example. It consists of an $\mathrm{Ar}^{+}$ laser with a coherence length of about $4 \mathrm{~cm}$, whose linear polarized beam was expanded to a diameter of about $2 \mathrm{~cm}$. The backscattered light was detected via a semi-transparent mirror by a CCD camera positioned in the focus of a 250 or $500 \mathrm{~mm}$ lens. Another linear polarizer (analyser) was placed between the semi-transparent mirror and the lens. Circular polarization was created (and detected) by placing a $\lambda / 4$ phase retarding plate between the semi-transparent mirror and the sample. The pictures of the camera, which are rotationally invariant in the case of circular polarization, were averaged in the azimuthal angle around the exact backscattering direction. In order to create a convergent or divergent wavefront, an additional lens was put between the semi-transparent mirror and the beam expander. The $7.5 \mathrm{~T}$ superconducting magnet had a horizontal bore with a diameter of $50 \mathrm{~mm}$ in which a non-depolarizing mirror was placed for illumination and detection perpendicular to $\vec{B}$. During a measurement cycle, the sample was moved to average out the speckles. If the sample consisted of many particles, the volume fraction was chosen such that multiple scattering by more than one sphere was negligible.

\section{Summary}

In sections 1 and 2 we gave a short summary of the present understanding of the 'glory' and $\mathrm{CB}$, putting emphasis on the comparison between the two phenomena. Both the 'glory' and $\mathrm{CB}$ are interference effects, scaling with $\lambda_{\mathrm{o}} / a$ and $\lambda_{\mathrm{o}} / \ell^{\star}$ depending on what the relevant parameter is. They originate from the symmetry around the exact backscattering direction (axial focusing). In the case of CB, there is only the symmetry between direct and reversed paths, leading to a twofold intensity enhancement, at most. The 'glory' is based on the interference between all paths which are rotationally invariant around the optical axis, leading to a destructive interference (i.e. no intensity) for $\theta_{b}=0$ in the case ' ++ ' and to an intensity maximum in the case ' +- '. We found that in the case ' ++ ' the oscillations of the 'glory' are well described by the square of the second-order Bessel function. The case '+-' corresponds to the square of a zeroth-order Bessel function but only on average over a certain size parameter range, which corresponds to a reduction of the coherence length. Note that sunlight (together with the sensitivity range of the eye) has a coherence length of approximately $2 \lambda^{2} / \pi \Delta \lambda=3 \mu \mathrm{m}$. By precisely studying both Bessel functions it turns out that the relevant wavelength for $m<\sqrt{ } 2$ is not $\lambda_{0}$ but the wavelength of the surface wave.

In section 3, we studied a sample of imperfect (not necessarily elliptic [21]) spheres whose backscattering pattern is proportional to $1+J_{0}\left(2 k_{0} a \theta_{b}\right)$. This corresponds to CB, i.e. solely to the interference between each light path and its reversed path. For further comparison we illuminated the samples with divergent or convergent wavefronts. In both cases, the scattering pattern reproduces in the plane around the (image) light source. Finally, we applied a magnetic field perpendicular to the incident light, resulting in a shift of the scattering pattern out of the exact backward direction due to magneto-optical Faraday rotation. This last experiment nicely demonstrates that it is an interference effect.

The 'glory' and CB are only two extreme cases of backscattering patterns. We have also observed situations lying in between. The comparison between the 'glory' and $\mathrm{CB}$ is important for the interpretation and evaluation of $\mathrm{CB}$ measurements in dense, disordered samples consisting of large particles, as there may be a smooth transition from interto intra-particle multiple scattering. These studies also put the meaning of 'single scattering' (which normally does not contribute to $\mathrm{CB}$ ) into another perspective. Finally, it is worth mentioning that a 'glory' measurement can be very useful for the alignment and calibration of a CB set-up. 


\section{Acknowledgments}

We would like to thank Ralf Tweer, Willy Leutz, Jaro Rička, Guido Bucher and Ivo Flammer for fruitful discussion as well as the supply of the glass beads.

\section{References}

[1] Nussenzveig H M 1992 Diffraction Effects in Semiclassical Scattering (Cambridge: Cambridge University Press)

[2] Bryant H C and Nelson 1974 The glory Sci. Am. July 60-71

[3] Fraser A B 1994 The sylvanshine: retroreflection from dew-covered trees Appl. Opt. 33 4539-47

[4] van Albada M P and Lagendijk A 1985 Observation of weak localization of light in a random medium Phys. Rev. Lett. 55 2692-5

[5] Wolf P E and Maret G 1985 Weak localization and coherent backscattering of photons in disordered media Phys. Rev. Lett. 55 2696-9

[6] Saxon D S 1995 Tensor scattering matrix for the electromagnetic field Phys. Rev. 100 1771-5

[7] Lenke R and Maret G 2000 Multiple scattering of light: coherent backscattering and transmission Scattering in Polymeric and Colloidal Systems ed W Brown and K Mortensen (London: Gordon and Breach) p 1

[8] van de Hulst H C 1957 Light Scattering by Small Particles (New York: Dover)

[9] Lock J A 1998 Cooperative effects among partial waves in Mie scattering J. Opt. Soc. Am. A 5 2032-44
[10] Fahlen T S and Bryant H C 1968 Optical back scattering from single water droplets J. Opt. Soc. Am. 58 304-10

[11] Hovenac E A and Lock J A 1992 Assessing the contribution of surface waves and complex rays to far-field Mie scattering by use of the Debye series J. Opt. Soc. Am. A 9 781-95

[12] Ashkin A and Dziedzic J M 1977 Observations of resonances in the radiation pressure on dielectric spheres Phys. Rev. Lett. 38 1351-4

[13] Patitsas A J 1972 Rainbows, glories, and the scalar field approach Can. J. Phys. 50 3172-83

[14] Gorriaz J, Horvath H and Rainmann G 1986 Influence of small color differences on the contrast threshold: its application to atmospheric visibility Appl. Opt. 25 2537-45

[15] Kubesh R J 1992 Computer display of chromaticity coordinates with rainbow as an example Am. J. Phys. 60 919-23

[16] Lee R L 1998 Mie theory, Airy theory, and the natural rainbow Appl. Opt. 37 1506-19

[17] Born M and Wolf E 1959 Principles of Optics (Oxford: Pergamon)

[18] Golubentsev A A 1984 Suppression of interference effects in multiple scattering of light Sov. Phys.-JETP 59 26-32

[19] Lenke R and Maret G 2000 Magnetic field effects on coherent backscattering of light Eur. J. Phys. B 17 171-85

[20] Lenke R, Lehner R and Maret G 2000 Magnetic-field effects on coherent backscattering of light in case of Mie spheres Europhys. Lett. 52 620-6

[21] Arnott W P and Marston P L 1991 Unfolded optical glory of spheroids: backscattering of laser light from freely rising spheroidal air bubbles in water Appl. Opt. 30 3429-42 